ISSN 1991-

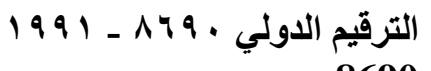

Website: http://jsci.utq.edu.iq

Email: utjsci@utq.edu.iq

\title{
Carrier Excitation Energies from a QD to OCL, RMS of Relative QD-Size Fluctuations and Temperature Dependence of QDL
}

\author{
$\begin{array}{lll}\text { R. M. Hassan } & \text { C. A. Emshary S. I. Easa }\end{array}$ \\ Dept. of Physics - College of Education - University of Basrah
}

\begin{abstract}
In this work, we study the characteristic temperature of a quantum dot laser (QDL) in presence of internal optical loss and quantum efficiency. The control parameters (the constant component of internal loss coefficient, effective cross section, carrier excitation energies from a QD to the optical confinement layer (OCL), and the root mean square (RMS) of relative QD-size fluctuations) are used for achieving free carrier density in the OCL and threshold current density and its component.
\end{abstract}

\section{Introduction}

The fluctuations in QD parameters lead to an inhomogeneously broadened gain spectrum [1]. Inhomogeneous line broadening and its effect on the threshold current of a QD laser were analyzed by $[1,2]$. As well as on other parameters, the dependence of the threshold current density on the root mean square of QD size fluctuations was calculated by $[3,4]$. In actual QD structures, there are also excited-states. In an ideal scenario, there should be one electron and one hole energy levels in a QD. The effect of excited-states on the threshold current density of a QD laser was studied [5]; a small overlap integral for transitions in QDs was shown to be a possible reason for a low single-layer modal gain limiting the ground-state lasing in short-cavity devices [6]. In Refs.[3,7,8] the charge neutrality violation in QDs and its effect on the laser characteristics were studies. It was shown that the electron and hole level occupancies in a QD can differ from each other, and the difference can be comparable to the occupancies themselves. The internal loss was shown to considerably narrow the region of tolerable structure parameters in which the lasing is attainable. The calculations suggested that the internal loss is likely to be another major limiting factor for lasing in short-cavity QD structures $[9,10]$. The carrier-density dependent internal loss acts as an additional source of the temperature-dependence of the threshold current. Multiple mode generation can be due to longitudinal Spatial Hole Burning (SHB), which is caused by non- uniformity of the stimulated recombination along the cavity $[11,12,13]$. Non-uniformity of stimulated recombination can lead to non-uniform carrier 
distributions.In QW or bulk-active-region lasers, non-uniform carrier distributions are effectively smoothed out by the diffusion.

In a QD laser, smoothing out non-uniform carrier distribution requires also the thermal escape of carriers from QDs to the optical confinement layer. It is the thermal escape to be the limiting factor [14]. Therefore, SHB is more strongly pronounced in QD lasers as compared to bulk or QW lasers. On the structure parameters, Ref.[14] using a linear approximation to calculate the multimode generation threshold and studied the dependence of this threshold. It was found that a decrease in the QD size dispersion considerably increases the relative multimode generation threshold [8].

\section{Free-Carrier Densities}

One can use the rate equations model to calculate the threshold current densities and output powers of longitudinal modes. The steady state rate equations for carriers confined in QDs, free carriers in the optical confinement layer, and photons. The threshold current density, $j_{\text {th }}$ should remain unchanged with temperature and the characteristic temperature should be infinitely high (in ideal QD laser) $[15,16]$. This would be the case if the overall injection current went into QDs, and the recombination current in QDs would be temperature-independent. In actual QD lasers, carriers are first injected from the cladding layers into the Optical Confinement Layer (OCL) (which includes the wetting layer), and then captured into QDs. The presence of carriers in the OCL results in recombination therein. Hence the recombination processes both in QDs and in the OCL are control in $\hat{j}_{\text {th }}$ and its $T$-dependence [1,3]:

$T_{c}=\frac{j_{t h}}{\frac{j_{D}}{T_{C}^{D}}+\frac{j_{L}}{T_{C}^{L}}}$

where $T_{C}$ is characteristic temperature of $\mathrm{QD}$ laser, $T_{C}^{D}$ and $T_{C}^{L}$ are defined similarly to $T_{C}$ but for $\hat{j}_{D}$ and $\hat{l}_{L}$ respectively. $\hat{j}_{D}$ and $\hat{l}_{L}$ are the components of $\hat{j}_{\mathrm{th}}\left(=\hat{j}_{D}+\hat{j}_{L}\right)$ associated with the recombination in QDs and in OCL respectively. Those components are given as $[1,3,17]$ :

$$
\begin{aligned}
& j_{D}=\frac{e S_{d} n_{D}^{e} n_{D}^{\mathbf{h}}}{\tau_{D}} \\
& j_{L}=e z_{O} \mathcal{R}_{\bar{O}}^{e} \bar{n}_{O}^{\mathbf{h}}
\end{aligned}
$$

where $n_{D}^{e_{,}, \mathrm{h}}$ are the confined-electron / hole level occupancies in QDs at the lasing threshold respectively, $\bar{n}_{O}^{e_{j} h}$ are the free-electron / hole densities in the OCL at the lasing threshold respectively, $S_{d}$ is the surface density of QDs, $\tau D$ is the spontaneous radiative recombination time in

QDs, zo is the OCL thickness and $\mathcal{R}$ is the radiative constant for the OCL material.

The carrier distribution below and at the lasing threshold is described by the equilibrium statistics (relatively high $T$ ).Thus, the T-dependence of free-carrier densities $\left({ }_{0}^{e_{0}, h}\right.$ ), have acts as the major source of such dependence of $\hat{j}_{\mathrm{th}}[14,2,3], \bar{n}_{O}^{e_{\mathrm{r}} \mathbf{h}}$ depend exponentially on $T$; 
$\bar{n}_{O}^{e_{j},}=\frac{\bar{n}_{O_{0}}^{e_{h} h} n_{D}^{e_{j} h}}{1-n_{D}^{e_{j} h}}$

where

$\bar{n}_{O_{\mathrm{o}}}^{e_{\mathrm{h}}}=\mathcal{g}_{e_{\mathrm{h}} \mathrm{h}}^{L} \operatorname{Exp}\left[-\frac{E_{e_{\mathrm{j}} \mathrm{h}}}{T}\right]$

$q_{e_{3} h}^{L}=2\left(\frac{m_{e_{y} h}^{L} T}{2 \pi \hbar^{2}}\right)^{\frac{3}{2}}$

Where $m_{e_{1, h}}^{L}$ are the electron/ hole effective masses in the OCL respectively, $T$ is the temperature measured in units of energy, $E_{e_{,}, \mathrm{h}}$ are the electron / hole excitation energies from a QD to the OCL (see Fig.1).
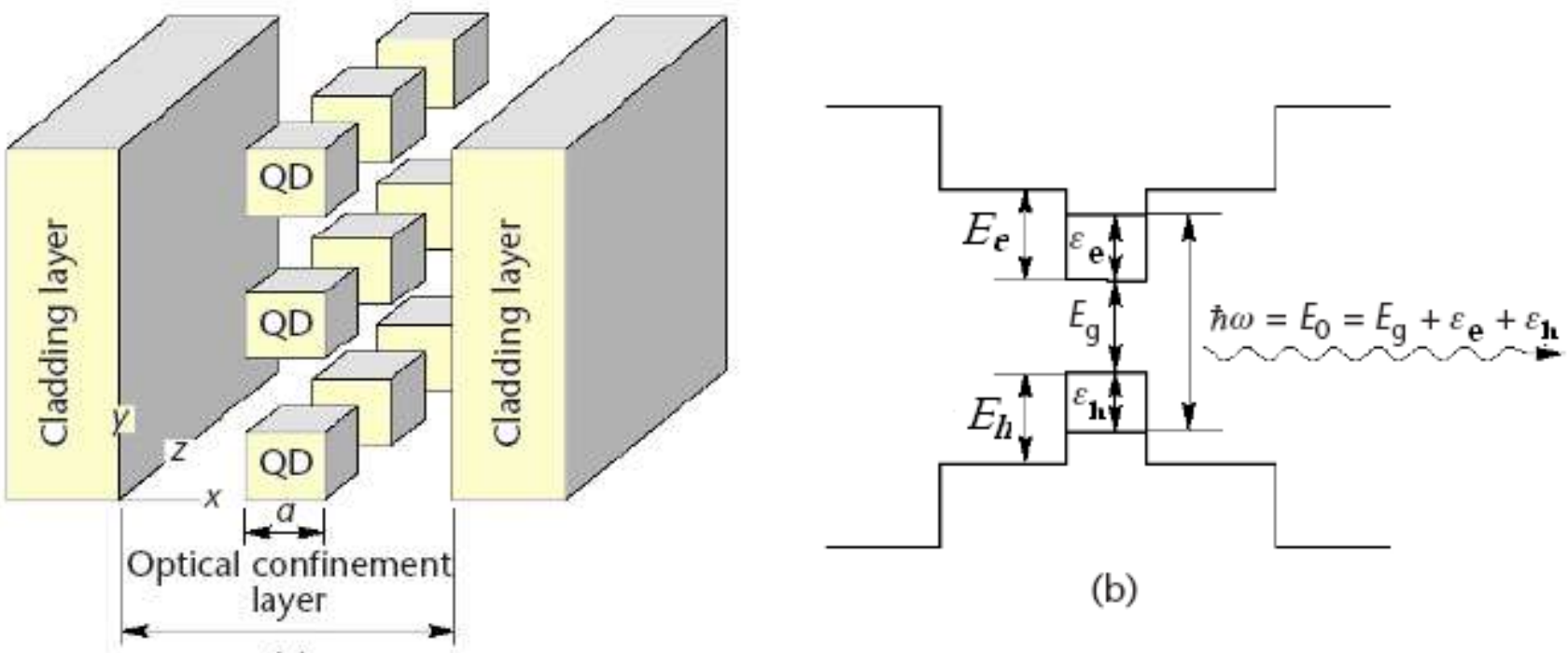

(b)

(a)

Figure 1: (a) Schematic structure and (b) energy band diagram of a QD [18].

$T$-dependence of the confined carrier level occupancies in QDs, $n_{D}^{e_{\text {, }}}$ can contributed different factors, thus causing the temperature-dependence of the recombination current density in QDs ( $\hat{l}_{D}$ ) and making $T_{C}^{D}$ finite $[19,20]$.

\section{QD Confined Carrier Occupancies}


As in other injection lasers, such a cavity loss can strongly affect the temperature stability of QD lasers [21], the effect of carrier-density-dependent internal optical loss in the OCL on the temperature dependence of $\hat{l}_{\text {th }}[10,17]$. To study the effect which is based on Ref.[9], where $\hat{l}_{\text {th }}$ has been calculated in the presence of the carrier-density-dependent internal loss. Neutrality, the charge is assumed hereto neatly clarify the effect of internal loss (in QDs $n_{D}^{e}=n_{D}^{h}$ and in the OCL $\bar{n}_{O}^{e}=\bar{n}_{O}^{\mathbf{h}}$ ) [22].

Such as free-carrier absorption in the OCL and scattering at rough surfaces and imperfections in the waveguide [9], several mechanisms can contribute to the internal loss. All these mechanisms can be conveniently grouped into two categories - one dependent on the carrier density in the OCL and the other independent. The expression for the saturation (maximum) value of the modal gain $G_{S}$ is [1]

$G_{S}=\frac{\varpi \hbar \Gamma S_{d}}{4 s_{m} \tau_{D}{x_{i n h}}_{i}}\left(\frac{\lambda}{\sqrt{U}}\right)^{2}$

where $\varpi$ is the QD-size distribution function $\left(\varpi=\frac{1}{\sqrt{2 \pi}}\right.$ for the Gaussian distribution), $\lambda$ is the wavelength of the main mode in vacuum, $\sqrt{U}$ is the group index of the dispersive OCL material, which is assumed to be the same for all modes, $\Gamma$ is the optical confinement factor in the QD layer, $S_{m}$ is the mean size of QDs and $\boldsymbol{L}_{i n h}$ is the inhomogeneous line broadening $[18,23,1]$,

$\varepsilon_{i n h}=\alpha\left(\Delta_{e} \varepsilon_{e}+\Delta_{h} \varepsilon_{h}\right)$

where $\alpha$ is RMS of relative QD-size fluctuations, $\varepsilon_{e_{\sharp} h}$ are the electron /hole quantized energy levels in a mean-sized QD (measured from the corresponding band edges),

$\Delta_{e, p}=-\frac{\partial \varepsilon_{e, p}}{\partial \ln S_{m}}$

The lasing threshold condition (equality of the gain to the loss) can be written as [7]

$G_{S}=\frac{M_{\text {loss }}+k_{i n}}{2 n_{D}^{e}-1}$

Where $M_{\text {loss }}$ is the mirror loss,

$M_{\text {loss }}=\frac{1}{\ell} \ln \left[\frac{1}{r}\right]$

Where $\ell$ is the cavity length and $r$ is the facet reflectivity. $k_{i n}$ is the overall internal loss coefficient which can be written as the sum of two components, one constant $\left(k_{C}\right)$ and the other increasing with the OCL carrier density $\left(\bar{n}_{0}^{e}\right)[9,10,17,24]$ 
$k_{\text {in }}=k_{c}+\mathcal{V}_{c s} \bar{n}_{O}^{e}$

Where $\nu_{C S}$ is the effective cross section for the internal absorption loss processes.

The level occupancy is immediately obtained from Eq.(10) to be independent of temperature, in the absence of the carrier-density-dependent internal loss ( $\left.\mathcal{V}_{C S}=0\right)$ [23],

$n_{D_{\mathrm{o}}}^{e}=\frac{1}{2}+\frac{k_{c}+M_{\text {loss }}}{2 G_{S}}$

The carrier-density-dependent internal loss couples $\bar{n}_{O}^{e}$ and $n_{D}^{e}$ and, as seen from Eq.(10), makes $n_{D}^{e}$ and $\hat{l}_{D}$ also temperature dependent, in view of the temperature dependence of $\bar{n}_{O}^{e}$. Thus, $T_{C}^{D}$ becomes finite. The expression for $n_{D}^{e}$ is easily found from Eqs.(4) and (10), which was derived in Ref.[9]

$n_{D}^{e}=\frac{1}{2}\left[\left(1+n_{D_{\mathrm{e}}}^{e}-\sigma_{1}\right)-\sqrt{\left(1+n_{D_{\mathrm{o}}}^{e}-\sigma_{1}\right)^{2}-4 n_{D_{\mathrm{e}}}^{e}}\right] \ldots(14)$

Where

$\sigma_{1}=\frac{\mathcal{V}_{C S} \bar{n}_{O_{\mathrm{e}}}^{e}}{2 G_{S}}$

\section{Free-carrier densities in the $\mathrm{OCL}$}

The free electron densities in the optical confinement layer have effects on the dynamics of QD laser. Returning back to the Eq.(4) and related equations it is so clear the effect of freeelectron densities in the OCL since Eq.(2) shows the dependence of threshold current component of QD on free-electron densities in the OCL, once more the effect of free-electron densities in the OCL is obvious on dynamics and power output of QD laser. To sum up the overall behavior effect of free-electron densities in the OCL with temperature for the same specific parameters mentioned: $k_{c}, V_{c s}$, and $E_{e}$ as shown in Fig.2.

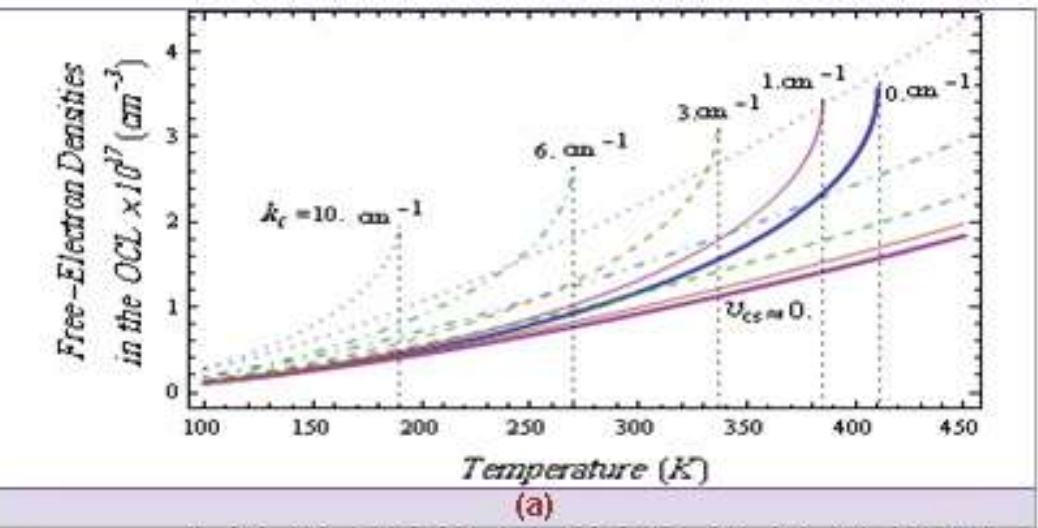


The striate line indicates $\bar{n}_{0}^{e}$ the absence of the carrier-density-dependent internal loss $\left(V_{C S} \approx 0\right)$. The vertical dotted line marks the certain temperature in the presence of the carrier density dependent internal loss. The other parameters [9,17]: $\mathrm{k}_{\mathrm{c}}=3 \mathrm{~cm}^{-1}, \mathrm{~s}_{\mathrm{m}}=150 \mathrm{~A}^{\mathrm{o}}, z_{\mathrm{o}}=0.28 \mu \mathrm{m}$, $\mathrm{S}_{\mathrm{d}}=6.11 \times 10^{10} \mathrm{~cm}^{-2}, \alpha=0.05, \mathcal{R}=20 . \times 10^{-30} \mathrm{~cm}^{3} \mathrm{~s}^{-1}, \tau_{\mathrm{D}}=1 . \mathrm{ns}, \mathrm{M}_{\text {loss }}=7 \mathrm{~cm}-1, \ell=1.628 \mathrm{~mm}, \mathrm{r}=0.32$, and $\Gamma=0.1$.

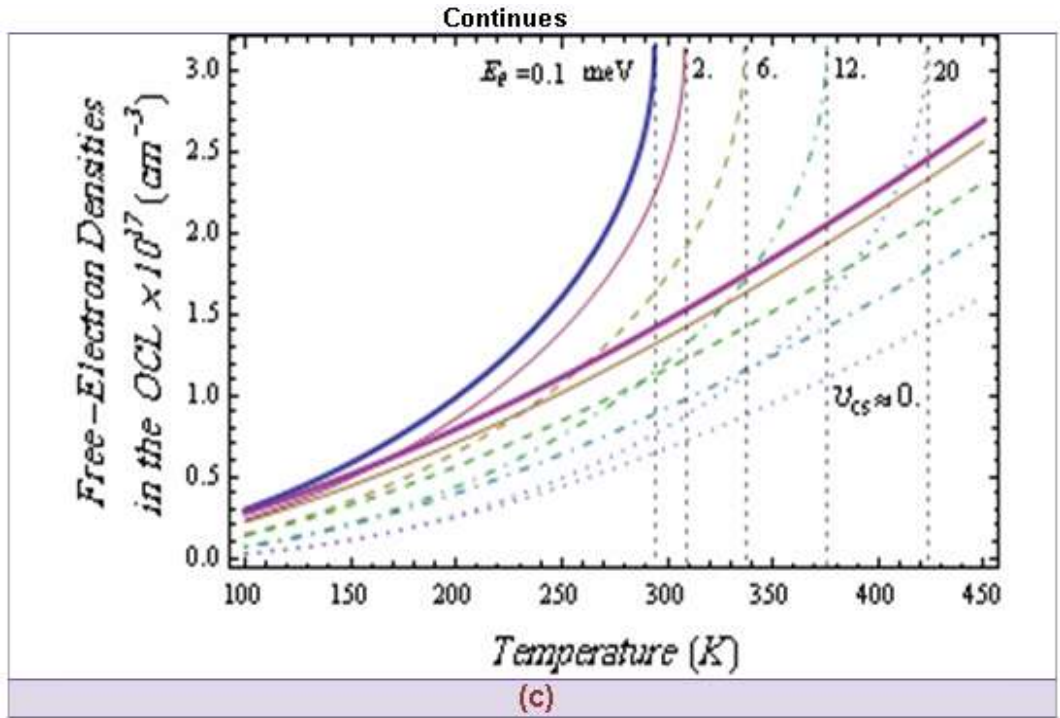




\section{Threshold Current Density}

A physical mechanism is proposed to explain why the sharp increase in the threshold current density at high temperatures is accompanied only by a relatively weak increase in its radiative component [25]. The radiative component of the threshold current density is stable to temperature relatively even when the threshold carrier density is increasing with temperature. This increasing carrier density, combined with the dominance of Auger recombination at $\mathrm{T}>200 \mathrm{~K}^{\mathrm{o}}$ [26] then gives rise to the strong temperature sensitivity of the threshold current density around room temperature in un-doped QD lasers.

As we have mentioned above the threshold current density is an important parameter in affecting the dynamics of semiconductor laser in general and QD laser in special since both in its two components $\hat{J}_{D}$ and $\hat{J}_{L}$ affect the $T_{C}$ which represents that limits for the possible use of QD laser.

The threshold current density affects the dynamics of QD lasers. Fig.3 examples of the obtained results for the radiation of threshold current density and its components, $\hat{J}_{D}$ and $j_{L}$ with temperature for the same different parameters used in the previous section.

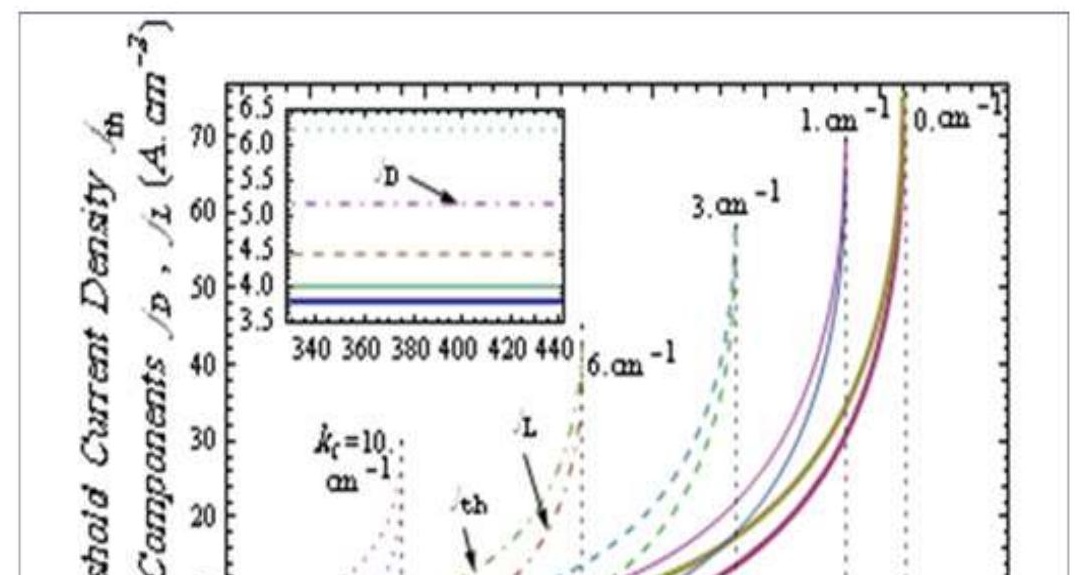


Continue

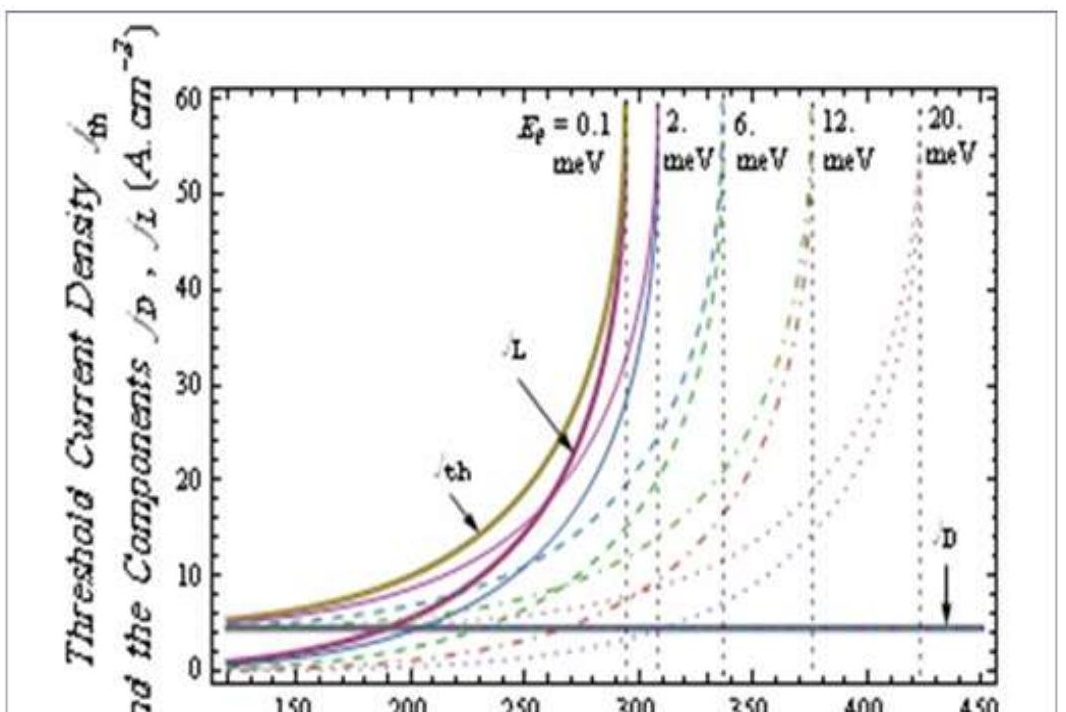




\section{Conclusion}

We studied limiting characteristics of a GaInAsP/InP QD laser with wavelength of $1.55 \mu \mathrm{m}$, that have been calculated as functions of temperature. The carrier-density-dependent internal loss in the OCL considerably reduces temperature. The internal loss in the optical confinement layer dependencies of different limits of QD laser characteristics has been studied. For a wide range of the structure parameters, the internal loss component is shown to play a main role in that effect, that increase with the OCL carrier density.

\section{Reference}

[1] L. V. Asryan and R. A. Suris, (1996). Semicond. Sci. Technol., 11(4): 554-567. 
[2] Levon V. Asryan and Serge Luryi, (2001). IEEE Journal of Quantum Electronics, 37 (7): 905 - 910.

[3] L. V. Asryan and R. A. Suris, (1998). IEEE Journal of Quantum Electronics, 34 (5): 841-850

[4] M.G. Thompson, K.T. Tan, C. Marinelli, K.A. Williams, R.V. Penty, I.H. White, M. Kuntz, D. Ouyang, D. Bimberg, V.M. Ustinov, A.E. Zhukov, A.R. Kovsh, N.N. Ledentsov, D.-J. Kang, and M.G. Blamire, (2004). Electronics Letters, 40(5): 346-347.

[5] Levon V. Asryan, Marius Grundmann, Nikolai N. Ledentsov, Oliver Stier, Robert A. Suris, and Dieter Bimberg, (2001). IEEE Journal of Quantum Electronics, 37(3):418-425.

[6] M. V. Maximov, L. V. Asryan, Yu. M. Shernyakov, A. F. Tsatsul'nikov, I. N. Kaiander, V. V. Nikolaev, A. R. Kovsh, S. S. Mikhrin, V. M. Ustinov, A. E. Zhukov, Zh. I. Alferov, N. N. Ledenstov, and D. Bimberg, (2001). IEEE Journal of Quantum Electronics, 37(5): 676-683.

[7] L. V. Asryan and R. A. Suris, (1997). IEEE Journal of Selected Topics in Quantum Electronics, 3(2):148-157 [8] L. V. Asryan and R. A. Suris, (2004). Semiconductors, 38(1): 1-22.

[9] Levon V. Asryan and Serge Luryi, (2004). IEEE Journal of Quantum Electronics, 40(7): 833-843.

[10] Levon V. Asryan, (2006). Applied Physics Latters, 88: 073107(1-3).

[11] N. F. Masse, I. P. Marko, A. R. Adams, and S. J. Sweeney, (2009). Journal of Mater Science: Mater Electron, 20: S272-S276.

[12] Pierre Meystre and Murray Sargent III, (2007). “Elements of Quantum Optics”. OSpringer-Verlag Berlin Heidelberg.

[13] Li Jiang and Levon V Asryan, (2008). Nanotechnology, 19: 415204(1-9).

[14] Levon V. Asryan and Robert A. Suris, (2000). IEEE Journal of Quantum Electronics,36(10): 11511160.

[15] Y. Arakawa and H. Sakaki, (1982), Applied Physics Letters, 40(11): 939-941.

[16] Kouichi Akahane, Naokatsu Yamamoto, and Tetsuya Kawanishi, (2010). IEEE Photonics Technology Letters, 22(2): 103-105.

[17] L. Jiang and L.V. Asryan. Laser Physics Letters, 4(4): 265-269, (2007).

[18] Todd Steiner, (2004). "Semiconductor Nanostructures for Optoelectronic Applications". (C) ARTECH HOUSE, INC.

[19] Dae-Seob Han and Levon V. Asryan, (2008). Solid-State Electronics, 52: 1674-1679.

[20] Dae-Seob Han and Levon V. Asryan, (2009). IEEE Journal of Lightwave Technology, 27(24):57755782.

[21] N.-H. Kim, J.-H. Park, L. J. Mawst, T. F. Kuech, and M. Kanskar, (2006). IEEE Photonics Technology Letters, 18(8): 989-991. 


\section{J.Thi-Qar Sci.}

[22] R Maram Q, H Baghban1, H Rasooli S, R Ghorbani, and A Rostami, (2009). Journal of Optics A: Pure and Applied Optics, 11: 105205(1-8).

[23] Li Jiang,(2008). PhD. thesis, Virginia Polytechnic Institute and State University, USA.

[24] Levon V. Asryan, (2006). Journal of Applied Physics, 99: 013102(1-4).

[25] Mark Thomas Crowley, Igor Pavlovich Marko, Nicolas F. Mass'e, Aleksey D. Andreev, Stanko Tomi'c, Stephen John Sweeney, Eoin P. O'Reilly, and Alfred R. Adams, (2009). IEEE Journal of Selected Topics in Quantum Electronics, 15(3): 799-807.

[26] I. P. Marko, N. F. Massé, S. J. Sweeney, A. D. Andreev, and A. R. Adams, (2005). Applied Physics Letters, 87:211114(1-3).

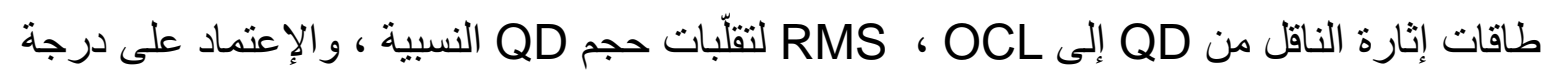
Q الحرارة في OLL

شاكر إبر اهيم عيسى

$$
\text { قاسب عبد الحسين مشاري }
$$$$
\text { رائد محمد حسن }
$$

\section{الخلاصة}

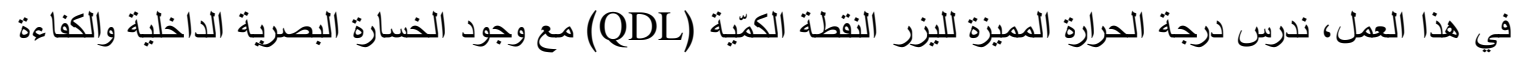

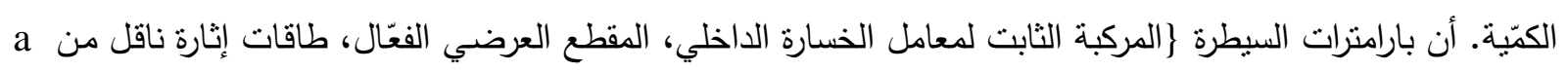

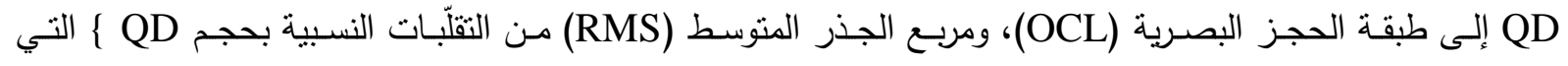
أستخدت لتحقيق كثافة الناقل الحرة في OCL وكثافة تيار العتبة ومكوّنها. 